\title{
A New Generalized Fractional-Order Derivative and Bifurcation Analysis of Cholera and Human Immunodeficiency Co-Infection Dynamic Transmission
}

\author{
Kumama Regassa Cheneke $\mathbb{D}^{D}$, Koya Purnachandra Rao ${ }^{(D)}$, and Gereme Kenassa Edesssa \\ Department of Mathematics, Wollega University, Nekemte, Ethiopia \\ Correspondence should be addressed to Kumama Regassa Cheneke; kumamaregassa@gmail.com
}

Received 23 October 2021; Accepted 24 January 2022; Published 23 February 2022

Academic Editor: Harvinder S. Sidhu

Copyright (c) 2022 Kumama Regassa Cheneke et al. This is an open access article distributed under the Creative Commons Attribution License, which permits unrestricted use, distribution, and reproduction in any medium, provided the original work is properly cited.

\begin{abstract}
In this study, the co-infection of HIV and cholera model has been developed and analyzed. The new fractional-order derivative is applied and the behavior of the solution is interpreted. The order of new generalized fractional-order derivative implication is presented. A new method is incorporated to determine the forward bifurcation at a threshold $R_{0}=1$. The developed method is used to determine the stability of steady-state points. The full model and the submodels' disease-free equilibrium are locally asymptotically stable if the corresponding reproduction number is less than one and unstable if the production number is greater than one. The only HIV model exhibits forward bifurcation at the threshold point, $R_{0}=1$. The numerical simulations solutions obtained using a new generalized fractional-order derivative shows that the total human population size approaches the diseasefree equilibrium if the order of the fractional derivative is higher. Also, the simulated results show that the memory effects toward the invading disease are less whenever the order of the fractional derivative is near 0 but higher whenever the order of the fractional derivative is near 1 . Furthermore, V. cholerae concentration in the environment increases whenever the intrinsic growth rate increases. The numerical solutions are carried out using MATLAB software.
\end{abstract}

\section{Introduction}

The individual infected with Human immunodeficiency virus (HIV) has a weak immune system [1-5]. HIV is the virus that invades targeted human T-cells directly $[6,7]$. The advanced stage HIV of causes the fatal disease called acquired immunodeficiency syndrome (AIDS) [5]. In 2018, globally the estimated number of people living with HIV was 37.9 million and estimated deaths were about 1.2 million. Moreover, only about $62 \%$ of HIV tested individuals were under ART [5]. Particularly, in Ethiopia, 2018, there were about 690 people living with HIV, about 23,000 were newly infected individuals, and 11000 people died because of infection [5]. The common modes of HIV transmission are unsafe sex, usage of HIV exposed materials, mother to child during pregnancy, and untested blood transfusion. Individuals who get infected with HIV/AIDS can be treated with antiretroviral therapy (ART) [5]. The individual who get cholera infection can be dead within an hour if not treated instantly [8]. The transmission ways of cholera infection is through eating food and drinking water exposed to Vibrio cholerae that causes the infection [9-14]. Cholera infected individuals can be treated with oral rehydration solution (ORS). HIV infected individuals has high susceptibility to cholera infection [4]. The individuals identified with cholera infection need to be tested for HIV infection.

The mathematical model is the widely used science to describe the dynamic behavior of population toward infections. For instance, a new generalized fractional order is applied in the epidemiology $[15,16]$. Moreover, mathematical models are widely applied in modeling different infections [16-30].

In this study, we are motivated by a new generalized fractional-order derivative described and applied in the 
recent works in $[15,16]$. Moreover, we have modified the works of [4], and a new generalized fractional-order derivative is applied to the developed HIV-cholera co-infection model.

\section{Mathematical Model}

In this study, we have developed the HIV and cholera coinfection model (Figure 1). We have divided the population targeted for this study into ten exclusive compartments which consist of the following. (i) Susceptible individuals $(S)$ : these are noninfectious individuals who can be infected with possible exposure to the infection. (ii) Only cholera-infected individuals $(C)$ : these are individuals infected with only cholera infection. (iii) Recovered individuals $(R)$ : these are recovered individuals who are infected by only cholera infection. (iv) HIV-infected individuals (I): these are individuals who get infected with HIV and at pre-AIDS stage. (v) HIV-cholera co-infected individuals $(D)$ : these are individuals who are infected both with HIV and cholera infection. They are at pre-AIDS stage. (vi) Recovered HIV individuals $(E)$ : these are HIV infected and cholera recovered individuals. They are at pre-AIDS stage. (vii) AIDS individuals $(A)$ : these are individuals who get infected with only HIV and at AIDS stage. (viii) AIDS-cholera-infected individuals $(F)$ : these are individuals at the AIDS stage and infected with cholera infection. (ix) Recovered AIDS individuals $(G)$ : these are cholera-recovered AIDS individuals. (x) Vibrio cholerae concentration $(B)$ : these are concentration of Vibrio cholerae in the environment that causes cholera infection with possible feeding the contaminated food or water.

Furthermore, the following assumptions are considered in the development of the HIV and cholera model:

(i) The population is assumed to be homogeneous.

(ii) Recruitment rate of individuals into susceptible compartment is $\tau$.

(iii) The total population size is nonconstant.

(iv) The total population size of human population under the study is denoted by $N_{H}(t)$ and defined as

$$
N_{H}(t)=S(t)+C(t)+R(t)+I(t)+D(t)+E(t)+A(t)+F(t)+G(t) .
$$

(v) All human populations die at natural death rate of $\mu$.

(vi) All individuals in $C$ and $D$ die due to cholera infection at the rate of $\varphi$ and $\psi$, respectively.

(vii) All individuals in $A, F$, and $G$ die at disease-induced death rate of $\delta, \varsigma$, and $\zeta$, respectively.

(viii) The transferring rate of $C$ to $R$ due to treatment is $\omega$.

(ix) The transferring rate of $D$ to $E$ due to treatment is $\kappa$.

(x) The transferring rate of $F$ to $G$ due to treatment is $\rho$.

(xi) The rate of losing immunity for $R, E$, and $G$ are $\gamma, \eta$, and $\phi$, respectively.

(xii) HIV is transmitted at transmission rate is $\beta_{h}$.

(xiii) Cholera infection transmission rate is $\beta$.

(xiv) Logistic growth model is considered for concentration of $V$. cholerae in the environment.

(xv) Intrinsic growth rate is $r$.

(xvi) Concentration of $V$. cholerae in the environment that causes $50 \%$ cholera infection is $\kappa$.

(xvii) $K$ is the carrying capacity of $V$. cholerae in the environment.

(xviii) $V$. cholerae die at the rate $\nu$.

The mathematical model of the HIV-cholera co-infection is given by

$$
\begin{aligned}
& \frac{\mathrm{d} S}{\mathrm{~d} t}=\tau-\left(\lambda_{1}+\lambda_{2}\right) S-\lambda_{3} S+\gamma R-\mu S, \\
& \frac{\mathrm{d} C}{\mathrm{~d} t}=\left(\lambda_{1}+\lambda_{2}\right) S-\lambda_{3} C-(\omega+\varphi+\mu) C, \\
& \frac{\mathrm{d} R}{\mathrm{~d} t}=\omega C-\lambda_{3} R-(\gamma+\mu) R, \\
& \frac{\mathrm{d} I}{\mathrm{~d} t}=\lambda_{3} S-\left(\lambda_{1}+\lambda_{2}\right) I+\eta E-(\alpha+\mu) I, \\
& \frac{\mathrm{d} D}{\mathrm{~d} t}=\left(\lambda_{1}+\lambda_{2}\right) I+\lambda_{3} C-(\psi+\xi+\kappa+\mu) D, \\
& \frac{\mathrm{d} E}{\mathrm{~d} t}=\lambda_{3} R+\kappa D-(\eta+\theta+\mu) E,
\end{aligned}
$$

$$
\begin{aligned}
& \frac{\mathrm{d} A}{\mathrm{~d} t}=\alpha I-\left(\lambda_{1}+\lambda_{2}\right) A+\phi G-(\delta+\mu) A, \\
& \frac{\mathrm{d} F}{\mathrm{~d} t}=\left(\lambda_{1}+\lambda_{2}\right) A+\xi D-(\rho+\varsigma+\mu) F, \\
& \frac{\mathrm{d} G}{\mathrm{~d} t}=\rho F+\theta E-(\phi+\zeta+\mu) G, \\
& \frac{\mathrm{d} B}{\mathrm{~d} t}=r B\left(1-\frac{B}{K}\right)-\nu B,
\end{aligned}
$$

with nonnegative initial conditions, where $\lambda_{1}=\beta_{c} B / k+B, \lambda_{2}=\beta(C+D+F)$, and $\lambda_{3}=\beta_{h}(I+D+E)$. 


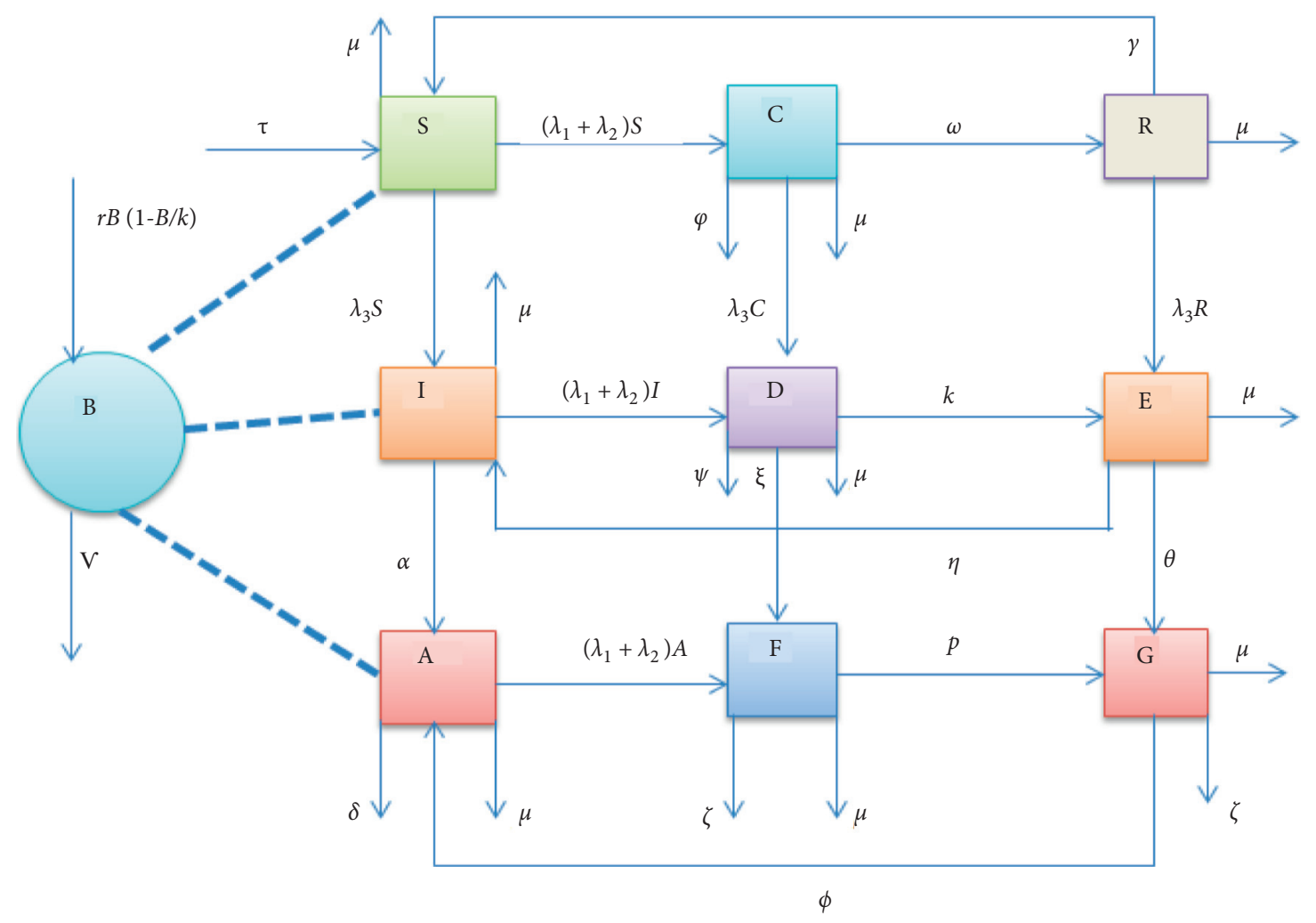

Figure 1: Flow diagram of HIV and cholera transmission dynamics.

\section{Mathematical Analysis of the Submodels}

3.1. Only HIV Model. In the absence of cholera, infection model (2) reduces to the form:

$$
\begin{aligned}
& \frac{\mathrm{d} S}{\mathrm{~d} t}=\tau-\beta_{h} I S-\mu S, \\
& \frac{\mathrm{d} I}{\mathrm{~d} t}=\beta_{h} I S-(\alpha+\mu) I, \\
& \frac{\mathrm{d} A}{\mathrm{~d} t}=\alpha I-(\delta+\mu) A,
\end{aligned}
$$

with nonnegative initial conditions.

\subsubsection{Invariant Region}

Theorem 1. The proper subset $\Omega$ of $\Re_{+}^{3}$ is a solution region of only the HIV model such that

$$
\Omega=\left\{(S, I, A) \in \mathfrak{R}_{+}^{3}: N_{h}=S+I+A \leq \frac{\tau}{\mu}\right\} .
$$

Proof. Adding left and right expressions of model (3), we obtain

$$
\frac{\mathrm{d} N_{h}}{\mathrm{~d} t}=\tau-\mu N_{h}-\delta A \leq \tau-\mu N_{h}
$$

Applying integration on the preceding inequality, we obtain

$$
N_{h} \leq \frac{\tau}{\mu}-\left(\frac{\tau}{\mu}-N_{h 0}\right) e^{-\mu t} .
$$

As time gets larger and larger, the preceding expression is bounded above by $\tau / \mu$. Therefore, the solutions of only the HIV model are bounded in the invariant $\Omega$ such that

$$
\Omega=\left\{(S, I, A) \in \mathfrak{R}_{+}^{3}: N_{h}=S+I+A \leq \frac{\tau}{\mu}\right\} .
$$

\subsubsection{Positivity Property}

Theorem 2. The solutions of only HIV model are nonnegative for all time $t$.

Proof. Considering the first equation of model (3), we obtain

$$
\frac{\mathrm{d} S}{\mathrm{~d} t}=\tau-\beta_{h} I S-\mu S
$$

Ignoring the positive term $\tau$, the forgoing equation reduces to the inequality of the form:

$$
\frac{\mathrm{d} S}{\mathrm{~d} t} \geq-\beta_{h} I S-\mu S
$$


Applying integration and solving for variable $S$, the foregoing inequality reduces to

$$
S(t) \geq S(0) e^{-\mu t-\beta_{h}} \int_{0}^{t} I(\xi) S(\xi) d \xi
$$

In the preceding inequality, $S(0)$ and $e^{-\mu t-\beta_{h} \int_{0}^{t} I(\xi) S(\xi) d \xi}$ are nonnegative for all time $t$. Therefore, $S(t)$ is nonnegative for all time $t$. Similarly, other state variables are nonnegative. Therefore, all solutions are nonnegative.

\subsubsection{Existence and Uniqueness of Solutions}

Proof. The expressions on the right of equality in the only HIV model are continuously differentiable everywhere. Therefore, the solutions of the only HIV model exist and are unique by Cauchy-Lipchitz theorem, stated in [11].

3.1.4. Disease-Free Equilibrium. The disease-free equilibrium $E_{0 h}$ is a steady state where there is no disease in the population. To obtain disease-free equilibrium, we set the rate of change of variables in model (3) equals to zero along with $I=A=0$. That is,

$$
\tau-\mu S=0 .
$$

$$
\frac{\mathrm{d}}{\mathrm{d} t}\left(\begin{array}{c}
I \\
A
\end{array}\right)=\left(\begin{array}{cc}
\beta_{h} S & 0 \\
0 & 0
\end{array}\right)\left(\begin{array}{c}
I \\
A
\end{array}\right)-\left(\begin{array}{cc}
\alpha+\mu & 0 \\
-\alpha & \delta+\mu
\end{array}\right)\left(\begin{array}{l}
I \\
A
\end{array}\right)=(F-V)\left(\begin{array}{l}
I \\
A
\end{array}\right) .
$$

Taking $F=\left(\begin{array}{cc}\beta_{h} S & 0 \\ 0 & 0\end{array}\right)$ and $V=\left(\begin{array}{cc}\alpha+\mu & 0 \\ -\alpha & \delta+\mu\end{array}\right)$, the next-generation matrix $F V^{-1}$ is given by

$F V^{-1}=\left(\begin{array}{cc}\beta_{h} S & 0 \\ 0 & 0\end{array}\right)\left(\begin{array}{cc}\alpha+\mu & 0 \\ -\alpha & \delta+\mu\end{array}\right)^{-1}=\left(\begin{array}{cc}\frac{\beta_{h} S}{\alpha+\mu} & 0 \\ 0 & 0\end{array}\right)$.

The eigenvalues of the foregoing next-generation matrix are given by

$$
\begin{aligned}
& \lambda_{1}=\frac{\beta_{h} S}{\alpha+\mu}, \\
& \lambda_{2}=0 .
\end{aligned}
$$

Therefore, the reproduction number $R_{0 h}=\rho\left(F V^{-1}\right)$, spectral radius of next-generation matrix, is given by

$$
R_{0 h}=\frac{\beta_{h} S^{0}}{\alpha+\mu}
$$

where $S^{0}=\tau / \mu$.
Hence, solving the equation, we obtain

$$
E_{0 h}=\left(\frac{\tau}{\mu}, 0,0\right) \text {. }
$$

3.1.5. Endemic Equilibrium. An endemic equilibrium $E_{h}=$ $\left(S^{*}, I^{*}, A^{*}\right)$ is a steady state at which the disease persists in the population. To obtain the endemic equilibrium, we set

$$
\begin{aligned}
\tau-\beta_{h} I S-\mu S & =0, \\
\beta_{h} I S-(\alpha+\mu) I & =0, \\
\alpha I-(\delta+\mu) A & =0 .
\end{aligned}
$$

Solving the foregoing equation, we obtain

$$
E_{h}=\left(\frac{\alpha+\mu}{\beta_{h}}, \frac{\mu\left(R_{0}-1\right)}{\beta_{h}}, \frac{\alpha \mu\left(R_{0}-1\right)}{\beta_{h}(\delta+\mu)}\right) \text {. }
$$

where $R_{0}=\tau \beta_{h} / \mu(\alpha+\mu)$.

3.1.6. Reproduction Number. The reproduction number $R_{0 h}$ is computed from the only HIV model using the nextgeneration method. Moreover, from thw only HIV model, the rate of change of infected variables can be written as
3.1.7. Bifurcation and Stability Analysis. Center manifold theory is widely used to determine the occurrence of forward bifurcation and backward bifurcation at equilibrium point. For instance, bifurcation analysis is performed in the works done in [28-35]. Moreover, based on application of center manifold theory, we have stated an efficient new generalized method to determine a kind of bifurcation that occurs at $R_{0}=1$ and stability behavior of equilibriums of the model without computing bifurcation parameters required in center manifold theory on bifurcation analysis.

Theorem 3 (new generalized method for bifurcation and stability analysis).

(i) Bifurcation diagram is an efficient diagram to determine the occurrence of backward and forward bifurcations at $R_{0}=1$. Hence, simulate endemic equilibrium versus reproduction number,

(ii) An endemic equilibrium of the epidemic model undergoes forward bifurcation at $R_{0}=1$ if stability behavior of model equilibriums interchanged at $R_{0}=1$. Moreover, endemic equilibrium of a model is stable only if $R_{0}>1$.

(iii) An endemic equilibrium of the epidemic model undergoes backward bifurcation at $R_{0}=1$ if 
stability behavior of a model equilibria share the same stability behavior if $R_{0}<1$.

(iv) In backward bifurcation, the endemic equilibrium exhibits both stable and unstable behavior for $R_{0}$ which is less than bifurcation parameter yielding point.

(v) An epidemic model with endemic equilibrium that undergoes forward bifurcation at $R_{0}=1$ has locally and globally asymptotically stable endemic equilibrium if $R_{0}>1$.

(vi) A model with endemic equilibrium that behaves forward bifurcation at $R_{0}=1$ has locally and globally asymptotically stable disease-free equilibrium if $R_{0} \leq 1$.

In this section, we simulate endemic equilibrium versus reproduction number to identify the kind of bifurcation that occurs at the point $R_{0}=1$ and to determine the stability behavior of equilibriums if $R_{0}<1$ and $R_{0}>1$.

Theorem 4. The only HIV model exhibits forward bifurcation at $R_{0}=1$ provided that only disease-free equilibrium is stable if $R_{0}<1$ and endemic equilibrium is only positively stable if $R_{0}>1$.

Proof. It follows from Figure 2.

\subsubsection{Local Stability of HIV-Free Equilibrium}

Theorem 5. The HIV-free equilibrium of the only HIV model is locally asymptotically stable if $R_{0}<1$ and unstable if $R_{0}>1$.

Proof. The Jacobian matrix $J$ constructed from the only $\mathrm{HIV}$ model at disease-free equilibrium is given by

$$
J=\left(\begin{array}{ccc}
-\mu & -\beta_{h} S^{0} & 0 \\
0 & \beta_{h} S^{0}-(\alpha+\mu) & 0 \\
0 & \alpha & -(\delta+\mu)
\end{array}\right) .
$$

The eigenvalues of the foregoing matrix are computed as

$$
\begin{aligned}
& \lambda_{1}=-\mu, \\
& \lambda_{2}=(\alpha+\mu)\left(R_{0 h}-1\right), \\
& \lambda_{3}=-(\delta+\mu) .
\end{aligned}
$$

Here, all computed eigenvalues are negative. Therefore, by stability theory of differential equations, the HIV-free equilibrium of the only HIV model is locally asymptotically stable if and only if $R_{0 h}<1$ and unstable if $R_{0 h}>1$.

\subsubsection{Global Stability of HIV-Free Equilibrium}

Theorem 6. The HIV-free equilibrium of the only HIV model is globally asymptotically stable if $R_{0}<1$ and unstable if $R_{0}>1$.

Proof. It follows from Theorem 3.

\subsubsection{Local Stability of Endemic Equilibrium}

Theorem 7. The HIV-free equilibrium of the only HIV model is locally asymptotically stable if $R_{0}>1$ and unstable if $R_{0}<1$.

Proof. It follows from Theorem 3.

\subsubsection{Local Stability of Endemic Equilibrium}

Theorem 8. The HIV-free equilibrium of the only HIV model is locally asymptotically stable if $R_{0}>1$ and unstable if $R_{0}<1$.

Proof. It follows from Theorem 3.

\subsubsection{Global Stability of Endemic Equilibrium}

Theorem 9. The endemic equilibrium of the only HIV model is globally asymptotically stable if $R_{0}>1$ and unstable if $R_{0}<1$.

Proof. It follows from Theorem 3.

3.2. Only Cholera Model. In the absence of HIV/AIDS in the population, the only cholera model is obtained from model (2) as

$$
\begin{aligned}
\frac{\mathrm{d} S}{\mathrm{~d} t} & =\tau-\left(\frac{\beta_{c} B}{k+B}+\beta C\right) S+\gamma R-\mu S . \\
\frac{\mathrm{d} C}{\mathrm{~d} t} & =\left(\frac{\beta_{c} B}{k+B}+\beta C\right) S-(\omega+\varphi+\mu) C, \\
\frac{\mathrm{d} R}{\mathrm{~d} t} & =\omega C-(\gamma+\mu), \\
\frac{\mathrm{d} B}{\mathrm{~d} t} & =r B\left(1-\frac{B}{K}\right)-\nu B,
\end{aligned}
$$

under nonnegative initial conditions.

\subsubsection{Invariant Region}

Theorem 10. The solutions of the only cholera model are invariant in the region $\Omega_{2}$ proper subset of $\Re_{+}^{4}$ such that $\Omega_{2}=\left\{(S, C, R, B): N_{c}=S+C+R<\tau / \mu, B \leq K\right\}$.

Proof. Adding the first three equations of the only cholera model, we obtain

$$
\frac{\mathrm{d} N_{c}}{\mathrm{~d} t}=\tau-\mu N_{c}-\varphi C \leq \tau-\mu N_{c} .
$$

Solving the above inequality over time interval $[0, t]$, we obtain

$$
N_{c} \leq \frac{\tau}{\mu}-\left(\frac{\tau}{\mu}-N_{c}(0)\right) e^{-\mu t}
$$




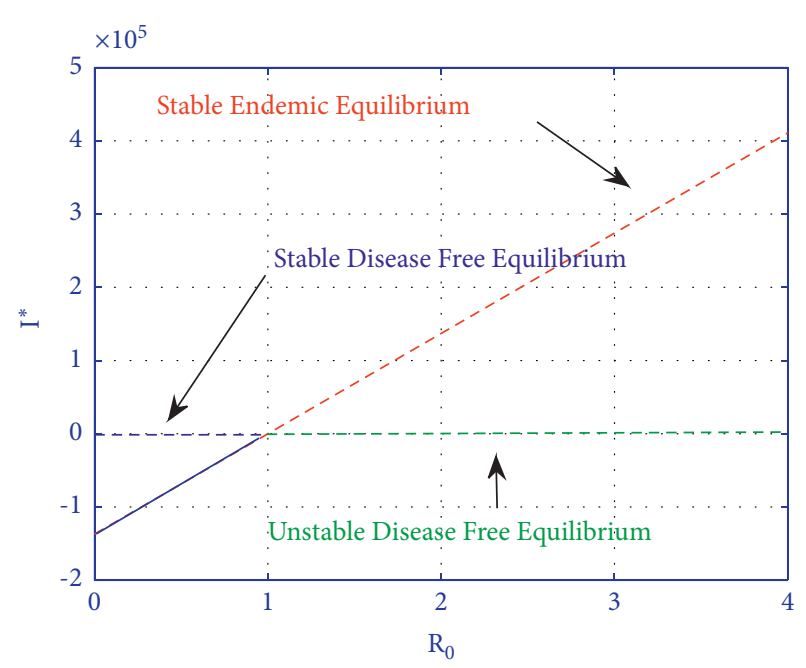

FIGURE 2: Illustration of forward bifurcation at $R_{0}=1$.

As time $t \longrightarrow \infty$, the total human population in the only cholera model $N_{c} \longrightarrow \tau / \mu$ for all time $t$. Also, from the last equation of the only cholera model, we have

$$
\frac{\mathrm{d} B}{\mathrm{~d} t} \leq r B\left(1-\frac{B}{K}\right)
$$

Solving the preceding inequality, we obtain

$$
\begin{aligned}
\left(\frac{1}{B}+\frac{1}{K-B}\right) \mathrm{d} B & \leq \frac{r}{K} \mathrm{~d} t, \\
\ln \frac{B(t)}{B(0)}-\ln \frac{K-B(t)}{K-B(0)} & \leq \frac{r}{K} \mathrm{~d} t, \\
B(t)+B(t)\left(\frac{B(0)}{K-B(0)}\right) e^{r t / K} & \leq K\left(\frac{B(0)}{K-B(0)}\right) e^{K / r t}, \\
B(t) & \leq \frac{K B(0)}{\left((K-B(0)) e^{-r t / K}+B(0)\right)} .
\end{aligned}
$$

As time $t \longrightarrow \infty, B(t) \longrightarrow K$. Thus, $B(t)$ is bounded above by carrying capacity of environment $K$. Hence, it completes the proof.

\subsubsection{Positivity Property}

Theorem 11. Solutions of the only cholera model are nonnegative for all time $t$.

Proof. The first equation of only cholera model (21) is given by

$$
\frac{\mathrm{d} S}{\mathrm{~d} t}=\tau-\frac{\beta_{c} B S}{k+B}-\beta S C+\gamma R-\mu S .
$$

Logically, ignoring the positive term $\tau+\gamma R$ in the foregoing equation, we obtain an inequality of the form:

$$
\frac{\mathrm{d} S}{\mathrm{~d} t} \geq-\frac{\beta_{c} B S}{k+B}-\beta S C-\mu S .
$$

Applying the integration and solving, we obtain

$$
S(t) \geq S(0) e^{-\mu t-\int_{0}^{t}\left(\left(\beta_{c} B(\xi) / k+B(\xi)\right)+\beta C(\xi)\right) d \xi} .
$$

Since $S(0)$ and the exponential expression $e^{-\mu t-\int_{0}^{t}\left(\beta_{c} B(\xi) / k+B(\xi)+\beta C(\xi)\right) \mathrm{d} \xi}$ is positive for all time $t$, the variable $S(t)$ is nonnegative for all time $t$.

\subsubsection{Existence and Uniqueness of Solutions}

Theorem 12. Solutions of only cholera model (21) exist and are unique.

Proof. The expressions on the right of only cholera model (21) are continuously differentiable and bounded. Therefore, the solutions of only cholera model (21) exist and are unique by Cauchy-Lipchitz theorem.

3.2.4. Cholera-Free Equilibrium. The cholera-free equilibrium of model (21) is computed by setting $C=B=0$ so that model (21) reduced to the form

$$
\begin{aligned}
\tau-\mu S & =0, \\
-(\gamma+\mu) R & =0 .
\end{aligned}
$$

Solving the preceding equation, we obtain

$$
\begin{aligned}
& S=\frac{\tau}{\mu}, \\
& R=0 .
\end{aligned}
$$

Therefore, cholera-free equilibrium is given by

$$
E_{0 c}=\left(\frac{\tau}{\mu}, 0,0,0\right)
$$

3.2.5. Endemic Equilibrium. The endemic equilibrium $E_{1}=$ $\left(S^{*}, C^{*}, R^{*}, B^{*}\right)$ of the only cholera model is computed by solving the equations:

$$
\begin{aligned}
\tau-\frac{\beta_{c} B S}{k+B}-\beta S C+\gamma R-\mu S & =0, \\
\frac{\beta_{c} B S}{k+B}+\beta S C-(\omega+\varphi+\mu) C & =0, \\
\omega C-(\gamma+\mu) R & =0, \\
r B\left(1-\frac{B}{K}\right)-\nu B & =0 .
\end{aligned}
$$

The computed endemic equilibrium $E_{1}=\left(S^{*} C^{*} R^{*} B^{*}\right)$ of the only cholera model is given by 


$$
E_{1}=\left(S^{*}, C^{*}, \frac{\omega}{\gamma+\mu} C^{*}, B^{*}\right) \text {. }
$$

where $I^{*}=\tau-\mu S^{*} / \omega+\varphi+\mu, B^{*}=K(1-\nu / r)=B$, and $S^{*}=S$ is the solution of the equation:

$$
\frac{\mu^{2} R_{0 c}}{\tau} S^{2}-\left(\frac{\beta_{c} B}{K+B}+\mu\left(R_{0 c}+1\right)\right) S+\tau=0 .
$$

3.2.6. Reproduction Number. The reproduction number is computed from model (21) using the next-generation method; by denoting the new infected individuals arrives at the class by $f$ and remaining terms by $v$, we obtain

$$
\begin{gathered}
f=\left(\begin{array}{c}
\frac{\beta_{c} B S}{k+B}+\beta S C \\
r B\left(1-\frac{B}{K}\right)
\end{array}\right), \\
v=\left(\begin{array}{c}
(\omega+\varphi+\mu) C \\
v B
\end{array}\right) .
\end{gathered}
$$

The constructed Jacobian matrices are

$$
\begin{aligned}
F & =\left(\begin{array}{cc}
\beta S \frac{\beta_{c} S}{k} \\
0 & r
\end{array}\right), \\
V & =\left(\begin{array}{cc}
\omega+\varphi+\mu & 0 \\
0 & v
\end{array}\right), \\
F V^{-1} & =\left(\begin{array}{cc}
\frac{\beta \tau}{\mu(\omega+\varphi+\mu)} & \frac{\beta_{c} \tau}{\mu k \nu} \\
0 & \frac{r}{\nu}
\end{array}\right) .
\end{aligned}
$$

Basically, the eigenvalues of the preceding next-generation matrix are $\lambda_{1}=\beta \tau / \mu(\omega+\varphi+\mu), \lambda_{2}=r / \nu$. Hence,

$$
R_{0 c}=\max \left\{\frac{r}{\nu}, \frac{\beta \tau}{\mu(\omega+\varphi+\mu)}\right\} .
$$

\subsubsection{Local Stability of Disease-Free Equilibrium}

Theorem 13. Cholerae-free equilibrium of the only cholera model is locally asymptotically stable if $R_{0}<1$ and unstable if $R_{0}>1$.

Proof. Similar to the works carried outin [15].

\section{Analysis of the Full Model}

4.1. Invariant Region
Theorem 14. The solutions of model (2) are invariant in the region $\Omega$, proper subset of eight-dimensional space over the set of nonnegative real numbers such that

$\Omega=\{(S, C, R, I, D, E, A, F, G, B):$

$\left.N_{h}=S+C+R+I+D+E+A+F+G \leq \frac{\tau}{\mu}, B \leq K\right\}$.

Proof. The proof of this theorem is similar to the proof done in only cholera model (21), to show the invariant region.

\subsection{Existence and Uniqueness of Solutions}

Theorem 15. The solutions of model (2) exist and are unique in the invariant region $\Omega \subset \mathfrak{R}_{+}^{8}$.

Proof. All expressions on the right-hand side of equality in model (2) are continuously differentiable. Therefore, by Cauchy-Lipchitz theorem stated, the solutions of model (2) exist and are unique.

4.3. Disease-Free Equilibrium. The disease-free equilibrium $E_{0}$ of model (2) is computed as

$$
E_{0}=\left(\frac{\tau}{\mu}, 0,0,0,0,0,0,0\right) .
$$

4.4. Reproduction Number. The next-generation method is applied to compute the reproduction number from model (2). Moreover, we defined the following vectors from model (2) logically as

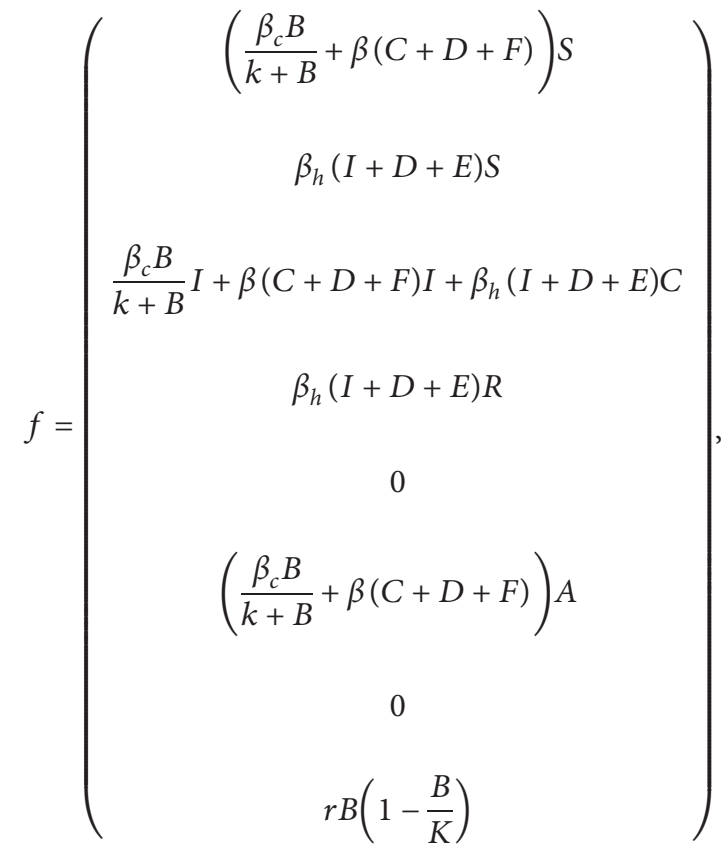




$$
\begin{aligned}
& v=\left(\begin{array}{c}
(\omega+\varphi+\mu) C \\
-\eta E+(\alpha+\mu) I \\
(\psi+\xi+\kappa+\mu) D \\
-\kappa D+(\eta+\theta+\mu) E \\
-\alpha I-\phi G+(\delta+\mu) A \\
-\xi D+(\rho+\varsigma+\mu) F \\
-\rho F-\theta E+(\phi+\zeta+\mu) G \\
\nu B
\end{array}\right) \\
& F=\left(\begin{array}{cccccccc}
\beta S & 0 & \beta S & 0 & 0 & \beta S & 0 & \left(\frac{S \beta_{c}}{k}\right) \\
0 & \beta_{h} S & \beta_{h} S & \beta_{h} S & 0 & 0 & 0 & 0 \\
0 & 0 & 0 & 0 & 0 & 0 & 0 & 0 \\
0 & 0 & 0 & 0 & 0 & 0 & 0 & 0 \\
0 & 0 & 0 & 0 & 0 & 0 & 0 & 0 \\
0 & 0 & 0 & 0 & 0 & 0 & 0 & 0 \\
0 & 0 & 0 & 0 & 0 & 0 & 0 & 0 \\
0 & 0 & 0 & 0 & 0 & 0 & 0 & r
\end{array}\right), \\
& V=\left(\begin{array}{cccccccc}
a & 0 & 0 & 0 & 0 & 0 & 0 & 0 \\
0 & \alpha+\mu & 0 & -\eta & 0 & 0 & 0 & 0 \\
0 & 0 & b & 0 & 0 & 0 & 0 & 0 \\
0 & 0 & -\kappa & c & 0 & 0 & 0 & 0 \\
0 & -\alpha & 0 & 0 & \delta+\mu & 0 & -\phi & 0 \\
0 & 0 & -\xi & 0 & 0 & d & 0 & 0 \\
0 & 0 & 0 & -\theta & 0 & -\rho & e & 0 \\
0 & 0 & 0 & 0 & 0 & 0 & 0 & \nu
\end{array}\right), \\
& F V^{-1}=\left(\begin{array}{cccccccc}
\frac{\beta S}{a} & 0 & \frac{\beta S}{b}+\frac{\beta \xi S}{d b} & 0 & 0 & \frac{\beta S}{d} & 0 & \frac{\beta_{c} \tau}{\mu k(\nu-r)} \\
0 & \frac{\beta_{h} S}{f} \frac{\beta_{h} S}{b}+\frac{\beta_{h} S \kappa}{c b}+\frac{\beta_{h} S \kappa \eta}{c b f} \frac{\beta_{h} S}{c}+\frac{\beta_{h} S \eta}{c f} & 0 & 0 & 0 & 0 \\
0 & 0 & 0 & 0 & 0 & 0 & 0 & 0 \\
0 & 0 & 0 & 0 & 0 & 0 & 0 & 0 \\
0 & 0 & 0 & 0 & 0 & 0 & 0 & 0 \\
0 & 0 & 0 & 0 & 0 & 0 & 0 & 0 \\
0 & 0 & 0 & 0 & 0 & 0 & 0 & 0 \\
0 & 0 & 0 & 0 & 0 & 0 & 0 & 0
\end{array}\right)
\end{aligned}
$$

where $a=\omega+\varphi+\mu, b=\psi+\xi+\kappa+\mu, c=\eta+\theta+\mu, d=\rho$ $+\varsigma+\mu, e=\phi+\zeta+\mu$, and $f=\alpha+\mu$. The foregoing nextgeneration matrix shows that the reproduction number produced by HIV infected individual is $\beta_{h} S / f$. The reproduction number produced by cholera-infected individual and environment is $\beta S / a$. Therefore, the basic reproduction number $R_{0}$ of the full model is given by

$$
R_{0}=\max \left\{R_{0 h}, R_{0 c}\right\} .
$$

4.5. Local Stability of Disease-Free Equilibrium of the Full Model
Theorem 16. The disease-free equilibrium of the co-infection model is locally asymptotically stable if $R_{0}<1$ and unstable if $R_{0}>1$.

Proof. To prove the theorem, we constructed a Jacobian matrix evaluated at disease-free equilibrium from the coinfection model as follows:

$$
\begin{aligned}
& J=\left(\begin{array}{cccccccccc}
-\mu & -\beta & \gamma & -\beta_{h} & -\beta-\beta_{h} & -\beta_{h} & 0 & -\beta & 0 & -\frac{\beta_{c}}{\kappa} \\
0 & \beta-a & 0 & 0 & \beta & 0 & 0 & \beta & 0 & \frac{\beta_{c}}{\kappa} \\
0 & \omega & -b & 0 & 0 & 0 & 0 & 0 & 0 & 0 \\
0 & 0 & 0 & \beta_{h}-c & \beta_{h} & \beta_{h}+\eta & 0 & 0 & 0 & 0 \\
0 & 0 & 0 & 0 & -d & 0 & 0 & 0 & 0 & 0 \\
0 & 0 & 0 & 0 & \kappa & -e & 0 & 0 & 0 & 0 \\
0 & 0 & 0 & \alpha & 0 & 0 & -f & 0 & \phi & 0 \\
0 & 0 & 0 & 0 & \xi & 0 & 0 & -g & 0 & 0 \\
0 & 0 & 0 & 0 & 0 & \theta & 0 & \rho & -h & 0 \\
0 & 0 & 0 & 0 & 0 & 0 & 0 & 0 & 0 & r-v
\end{array}\right) \\
& a=\omega+\varphi+\mu, \\
& b=\gamma+\mu, \\
& c=(\alpha+\mu), \\
& d=(\psi+\xi+\kappa+\mu), \\
& e=(\eta+\theta+\mu), \\
& f=(\delta+\mu), \\
& g=(\rho+\varsigma+\mu), \\
& h=(\phi+\zeta+\mu) \text {. }
\end{aligned}
$$

The computed eigenvalues of the preceding Jacobian matrix are

$$
\begin{aligned}
& \lambda_{1}=-\mu, \\
& \lambda_{2}=r-v, \\
& \lambda_{3}=a\left(R_{0 c}-1\right), \\
& \lambda_{4}=-b, \\
& \lambda_{5}=-d, \\
& \lambda_{6}=-e, \\
& \lambda_{7}=-f, \\
& \lambda_{8}=-g,
\end{aligned}
$$




$$
\begin{aligned}
\lambda_{9} & =-h, \\
\lambda_{10} & =c\left(R_{0 h}-1\right) .
\end{aligned}
$$

Here, all computed eigenvalues are negative if $r<v, R_{0 c}<1$, and $R_{0 h}<1$. Therefore, by stability theory, the disease-free equilibrium of the full model is locally asymptotically stable if $R_{0}<1$ and unstable if $R_{0}>1$.

\section{Global Stability of Disease-Free Equilibrium}

Theorem 17. A disease-free equilibrium of a full model is globally asymptotically stable if $0<R_{0}<1$ and otherwise unstable.

Proof. Let $X=(S, R) \in \mathfrak{R}_{+}^{2}$ represent uninfected individuals in the full model and $Y=(C I, D, E, A, F, G, B) \in \mathfrak{R}_{+}^{8}$ be individuals in the infected class. Hence, we write full model (2) as

$$
\begin{aligned}
\frac{\mathrm{d} X}{\mathrm{~d} t} & =H(X, Y), \\
\frac{\mathrm{d} Y}{\mathrm{~d} t} & =G(X, Y), \\
G(X, 0) & =0 .
\end{aligned}
$$

The disease-free equilibrium of the foregoing system is computed as

$$
E_{0}^{1}=\left(X^{0}, 0\right)
$$

To guarantee global stability, the method we applied must met conditions $H_{1}$ and $H_{2}$ as stated below:

$H_{1}$ : for $\mathrm{d} X / \mathrm{d} t=H(X, 0), X^{0}$ is globally asymptotically stable

$$
\begin{aligned}
& H_{2}: G(X, Y)=P Y-\widehat{G}(X, Y), \widehat{G}(X, Y) \geq 0 \text { for }(X, Y) \\
& \in \Omega
\end{aligned}
$$

Here, $P=D_{Y} G(X, 0)$ be Metzler matrix and $\Omega$ is a region where solutions are acceptable.

Considering the state variable of noninfected compartment in model (2), we have

$$
\frac{\mathrm{d} X}{\mathrm{~d} t}=\left(\begin{array}{c}
\frac{\mathrm{d} S}{\mathrm{~d} t} \\
\frac{\mathrm{d} R}{\mathrm{~d} t}
\end{array}\right)=\left(\begin{array}{c}
\tau-\left(\lambda_{1}+\lambda_{2}\right) S-\lambda_{3} S+\gamma R-\mu S \\
\omega C-\lambda_{3} R-(\gamma+\mu) R
\end{array}\right)=H(X, Y) .
$$

Now, setting

$$
H(X, 0)=0
$$

and solving, for $X=(S, R)$, we obtain

$$
X=\left(\frac{\lambda}{\mu}, 0\right) \text {. }
$$

Hence,

$$
X^{0}=\left(\frac{\lambda}{\mu}, 0\right) \text {. }
$$

As $t \longrightarrow \infty$, we observe that $X=S \longrightarrow \lambda / \mu$. Thus, $X^{0}$ is globally asymptotically stable equilibrium of the subsequent equation of the form:

$$
\frac{\mathrm{d} X}{\mathrm{~d} t}=H(X, 0)
$$

Furthermore, considering the state variable of the infected compartments in model (2), we have

$$
\begin{aligned}
\frac{\mathrm{d} C}{\mathrm{~d} t} & =\left(\frac{\beta_{c} B}{k+B}+\beta(C+D+F)\right) S-\beta_{h}(I+D+E) C-(\omega+\varphi+\mu) C, \\
\frac{\mathrm{d} I}{\mathrm{~d} t} & =\beta_{h}(I+D+E) S-\left(\frac{\beta_{c} B}{k+B}+\beta(C+D+F)\right) I+\eta E-(\alpha+\mu) I, \\
\frac{\mathrm{d} D}{\mathrm{~d} t} & =\left(\frac{\beta_{c} B}{k+B}+\beta(C+D+F)\right) I+\beta_{h}(I+D+E) C-(\psi+\xi+\kappa+\mu) D, \\
\frac{\mathrm{d} E}{\mathrm{~d} t} & =\beta_{h}(I+D+E) R+\kappa D-(\eta+\theta+\mu) E, \\
\frac{\mathrm{d} A}{\mathrm{~d} t} & =\alpha I-\left(\frac{\beta_{c} B}{k+B}+\beta(C+D+F)\right) A+\phi G-(\delta+\mu) A,
\end{aligned}
$$




$$
\begin{aligned}
& \frac{\mathrm{d} F}{\mathrm{~d} t}=\left(\frac{\beta_{c} B}{k+B}+\beta(C+D+F)\right) A+\xi D-(\rho+\varsigma+\mu) F, \\
& \frac{\mathrm{d} G}{\mathrm{~d} t}=\rho F+\theta E-(\phi+\zeta+\mu) G, \\
& \frac{\mathrm{d} B}{\mathrm{~d} t}=r B\left(1-\frac{B}{K}\right)-v B .
\end{aligned}
$$

Also, at disease-free equilibrium, the foregoing equations can be written as

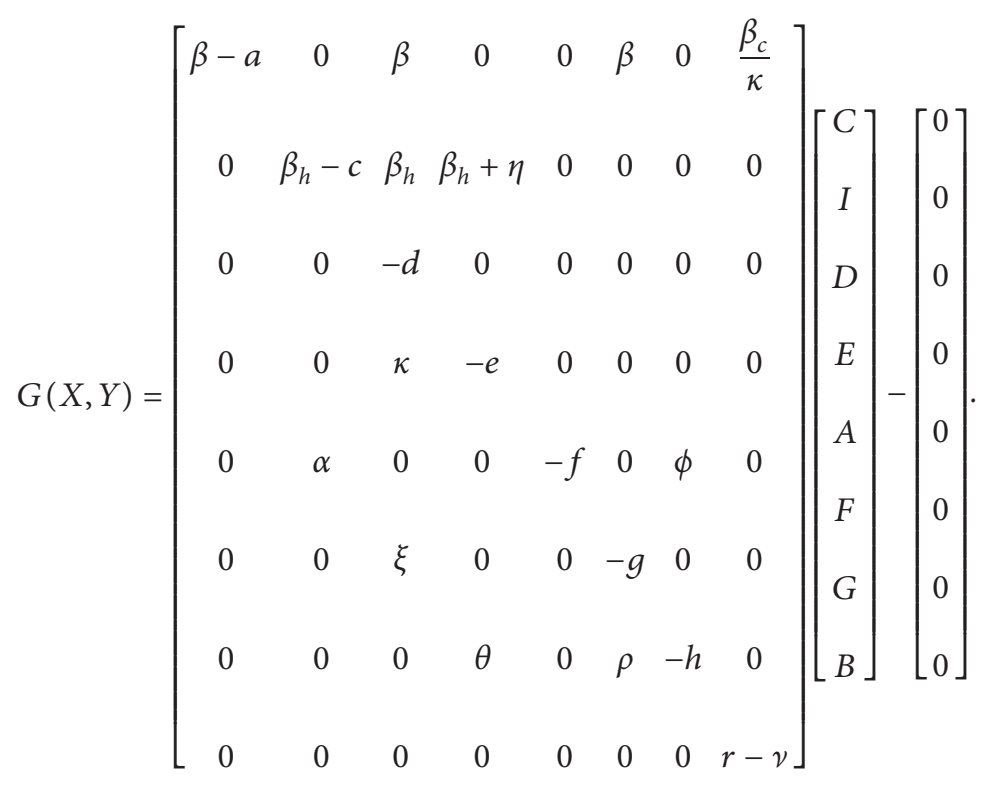

Now, comparing the required condition and the preceding equation, we obtain

$$
P=\left[\begin{array}{cccccccc}
\beta-a & 0 & \beta & 0 & 0 & \beta & 0 & \frac{\beta_{c}}{\kappa} \\
0 & \beta_{h}-c & \beta_{h} & \beta_{h}+\eta & 0 & 0 & 0 & 0 \\
0 & 0 & -d & 0 & 0 & 0 & 0 & 0 \\
0 & 0 & \kappa & -e & 0 & 0 & 0 & 0 \\
0 & \alpha & 0 & 0 & -f & 0 & \phi & 0 \\
0 & 0 & \xi & 0 & 0 & -g & 0 & 0 \\
0 & 0 & 0 & \theta & 0 & \rho & -h & 0 \\
0 & 0 & 0 & 0 & 0 & 0 & 0 & r-\nu
\end{array}\right],
$$


Now, we observe that $G(X, Y)=P Y-\widehat{G}(X, Y)$, where $\widehat{G}(X, Y) \geq 0, \forall X, Y$. Moreover, $P$ is Metzler matrix. Therefore, a disease-free equilibrium $E_{0}$ is globally asymptotically stable if $R_{0}<1$.

\section{A New Generalized Fractional- Order Derivative}

In this section, we apply a new generalized fractional-order derivative using the works in $[15,16]$. We study the dynamic behavior of the population study using a new generalized definition of derivative with fractional order.

First, let us consider only the rate of change of human population, from model (2), that can be described as

$$
\begin{aligned}
& \frac{\mathrm{d} S}{\mathrm{~d} t}=\tau-\left(\lambda_{1}+\lambda_{2}\right) S-\lambda_{3} S+\gamma R-\mu S, \\
& \frac{\mathrm{d} C}{\mathrm{~d} t}=\left(\lambda_{1}+\lambda_{2}\right) S-\lambda_{3} C-(\omega+\varphi+\mu) C, \\
& \frac{\mathrm{d} R}{\mathrm{~d} t}=\omega C-\lambda_{3} R-(\gamma+\mu) R, \\
& \frac{\mathrm{d} I}{\mathrm{~d} t}=\lambda_{3} S-\left(\lambda_{1}+\lambda_{2}\right) I+\eta E-(\alpha+\mu) I, \\
& \frac{\mathrm{d} D}{\mathrm{~d} t}=\left(\lambda_{1}+\lambda_{2}\right) I+\lambda_{3} C-(\psi+\xi+\kappa+\mu) D, \\
& \frac{\mathrm{d} E}{\mathrm{~d} t}=\lambda_{3} R+\kappa D-(\eta+\theta+\mu) E, \\
& \frac{\mathrm{d} A}{\mathrm{~d} t}=\alpha I-\left(\lambda_{1}+\lambda_{2}\right) A+\phi G-(\delta+\mu) A, \\
& \frac{\mathrm{d} F}{\mathrm{~d} t}=\left(\lambda_{1}+\lambda_{2}\right) A+\xi D-(\rho+\varsigma+\mu) F, \\
& \frac{d G}{d t}=\rho F+\theta E-(\phi+\zeta+\mu) G, \\
&
\end{aligned}
$$

with nonnegative initial conditions, where $\lambda_{1}=\beta_{c} B / k+B, \lambda_{2}=\beta(C+D+F)$, and $\lambda_{3}=\beta_{h}(I+D+E)$.

Let $N_{H}(t)$ be the size of human populations at time $t$ such that

$N_{H}(t)=S(t)+C(t)+R(t)+I(t)+D(t)+E(t)+A(t)+F(t)+G(t)$.
Differentiating both sides of the foregoing equation over time interval $[0, t]$ and using (54), we obtain

$\frac{\mathrm{d} N_{H}}{\mathrm{~d} t}=\tau-\mu \mathrm{N}_{\mathrm{h}}-\varphi \mathrm{I}-\psi \mathrm{D}-\delta \mathrm{A}-\varsigma \mathrm{F}-\zeta \mathrm{G} \leq \tau-\mu \mathrm{N}_{\mathrm{h}}$.

Now, considering the upper bound, the preceding inequality reduces to the form

$$
\frac{\mathrm{d} N_{H}}{\mathrm{~d} t}=\tau-\mu \mathrm{N}_{\mathrm{H}} .
$$

The solution of the preceding equation over time interval $[0, t]$ is given by

$$
N_{H}=\frac{\tau}{\mu}-\left(\frac{\tau}{\mu}-N_{H}(0)\right) e^{-\mu t}
$$

Definition 1 (a new generalized fractional derivative $[15,16])$. Let $\alpha \in[0,1), \beta, \gamma>0$, and $g \in H^{1}(a, b)$. In the sense given by Caputo, the $\alpha$ order constructed new generalized fractional derivative of some function $g(t)$ with respect to the weight function $w(t)$ is defined as

${ }^{C} D_{a, t, w}^{\alpha, \beta, \gamma} g(t)=\frac{N(\alpha)}{1-\alpha} \frac{1}{w(t)} \int_{a}^{t} E_{\beta}\left[-\mu_{\alpha}(t-x)^{\gamma}\right] \frac{\mathrm{d}}{\mathrm{d} x}(w g)(x) \mathrm{d} x$,

where $w \in C^{1}(a, b), w, w \prime>0$ on $[a, b], N(\alpha)$ is defined as a normalized function that satisfy the conditions $N(0)=N(1)=1, \mu_{\alpha}=\alpha / 1-\alpha, \quad$ and $\quad E_{\beta}(t)=\sum_{k=0}^{+\infty} t^{k}$ $/ \Gamma(\beta k+1)$ is the Mittag-Leffler function of parameter $\beta$. For simplicity, we symbolize ${ }^{C} D_{a, t, w}^{\alpha, \beta, \gamma} g(t)$ by $D_{a, t, w}^{\alpha, \beta, \gamma} g(t)$. Assuming that when HIV and cholera infections spread within a population, the individuals get knowledge about the infection; we replace the applied classical derivative by $D_{a, t, w}^{\alpha, \beta, \gamma}$. Then, (57) becomes

$$
D_{a, t, w}^{\alpha, \beta, \gamma} N_{h}(t)=\tau-\mu \mathrm{N}_{\mathrm{h}}(\mathrm{t}) .
$$

The basic properties of a new generalized fractional derivative are shown in [36]. Applying the Laplace transform on both sides of the foregoing equation, we obtain

$$
\mathscr{L}\left\{w(t) D_{a, t, w}^{\alpha, \beta, \gamma} N_{H}(t)\right\}=\tau \mathscr{L}\{w(t)\}-\mu \mathscr{L}\left\{w(t) N_{H}(t)\right\} .
$$

Using Theorem 2 [19], we have

$$
\mathscr{L}\left\{w(t) N_{H}(t)\right\}=\frac{N(\alpha) w(0) T(0) s^{\beta-1}}{[N(\alpha)+\mu(1-\alpha)] s^{\beta}+\alpha \mu}+\frac{\tau(1-\alpha) s^{\beta}+\lambda \alpha}{[N(\alpha)+\mu(1-\alpha)] s^{\beta}+\alpha \mu} \mathscr{L}\{w(t)\}
$$

Let $a_{\alpha}=N(\alpha)+\mu(1-\alpha)$. Then, 
$\mathscr{L}\left\{w(t) N_{H}(t)\right\}=\frac{N(\alpha) w(0) N_{H}(0)}{\alpha_{\alpha}} \frac{s^{\beta-1}}{s^{\beta}+\left(\alpha \mu / a_{\alpha}\right)}+\frac{\tau(1-\alpha)}{a_{\alpha}} \frac{s^{\beta-1}}{s^{\beta}+\left(\alpha \mu / a_{\alpha}\right)} s \mathscr{L}\{w(t)\}+\frac{\tau \alpha}{a_{\alpha}} \frac{1}{s^{\beta}+\left(\alpha \mu / a_{\alpha}\right)} \mathscr{L}\{w(t)\}$.

Therefore,

$\mathscr{L}\left\{w(t) N_{H}(t)\right\}=\frac{N(\alpha) w(0) N_{H}(0)}{\alpha_{\alpha}} \mathscr{L}\left\{E_{\beta}\left(-\frac{\alpha \mu}{a_{\alpha}} t^{\beta}\right)\right\}+\frac{\tau(1-\alpha)}{a_{\alpha}} \mathscr{L}\left\{E_{\beta}\left(-\frac{\alpha \mu}{a_{\alpha}} t^{\beta}\right)\right\}(\mathscr{L}\{w \prime(t)\}+w(0))-\frac{\lambda}{\mu} \mathscr{L}\left\{\frac{d}{d t} E_{\beta}\left(-\frac{\alpha \mu}{a_{\alpha}} t^{\beta}\right)\right\} \mathscr{L}\{w(t)\}$.

Applying inverse Laplace, the preceding equation reduces to

$w(t) N_{H}(t)=\frac{N(\alpha) w(0) N_{H}(0)}{\alpha_{\alpha}} E_{\beta}\left(-\frac{\alpha \mu}{a_{\alpha}} t^{\beta}\right)+\frac{\tau(1-\alpha)}{a_{\alpha}} E_{\beta}\left(-\frac{\alpha \mu}{a_{\alpha}} t^{\beta}\right) w^{\prime}(t)-\frac{\tau}{\mu} \frac{\mathrm{d}}{\mathrm{d} t} E_{\beta}\left(-\frac{\alpha \mu}{a_{\alpha}} t^{\beta}\right) w(t)+\frac{\tau(1-\alpha) w(0)}{a_{\alpha}} E_{\beta}\left(-\frac{\alpha \mu}{a_{\alpha}} t^{\beta}\right)$.

Letting $w(t)=1$ and applying the integration by parts, the preceding equation reduces to

$$
N_{H}(t)=\frac{\tau}{\mu}-\frac{N(\alpha)}{a_{\alpha}}\left(\frac{\tau}{\mu}-N_{H}(0)\right) E_{\beta}\left(-\frac{\alpha \mu}{a_{\alpha}} t^{\beta}\right) .
$$

In Section 7, using numerical simulations, we will analyze the impact of order of new fractional derivative on the behavior of solutions of the HIV and cholera co-infection model.

Next, we consider the rate of change of $V$. cholerae population, from model (2), that can be described as

$$
\frac{\mathrm{d} B}{\mathrm{~d} t}=r B\left(1-\frac{B}{K}\right)-v B,
$$

under nonnegative initial condition.

To get the size of $V$. cholerae populations $B(t)$ at time $t$, we set and solve (57) as follows:

$$
\frac{\mathrm{d} B}{\mathrm{~d} t}=r B\left(1-\frac{B}{K}\right)-v B .
$$

We rewrite the foregoing equation in the form

$$
\frac{\mathrm{d} B}{\mathrm{~d} t}=r B\left(\frac{(1-v) K-B}{K}\right) .
$$

Rearranging the terms, the foregoing equation reduces to the form

$$
\frac{\mathrm{d} B}{B((1-v) K-B)}=\frac{r}{K} \mathrm{~d} t .
$$

Integrating both sides of the preceding equation over time interval $[0, t]$, we obtain

$$
\int_{0}^{t} \frac{\mathrm{d} B}{B((1-\nu) K-B)}=\int_{0}^{t} \frac{r}{K} \mathrm{~d} t .
$$

Applying partial decomposition, we obtain

$$
\int_{0}^{t} \frac{1}{(1-v) K}\left(\frac{1}{B}+\frac{1}{(1-v) K-B}\right) \mathrm{d} B=\int_{0}^{t} \frac{r}{K} \mathrm{~d} t .
$$

Then, the computation of the preceding integral gives

$$
\ln \left|\frac{B(t)}{(1-v) K-B(t)} \times \frac{(1-v) K-B(0)}{B(0)}\right|=(1-v) r t .
$$

Now, applying the logarithmic property, the preceding equation reduces to

$$
\left|\frac{B(t)}{(1-v) K-B(t)} \times \frac{(1-v) K-B(0)}{B(0)}\right|=e^{(1-v) r t} .
$$
obtain

Rearranging the terms of the preceding equation, we

$$
\frac{B(t)}{(1-\nu) K-B(t)}=\frac{B(0) e^{(1-v) r t}}{(1-\nu) K-B(0)} .
$$

This implies

$$
B(t)=((1-v) K-B(t)) \frac{B(0) e^{(1-v) r t}}{(1-v) K-B(0)} .
$$

It follows that

$$
B(t)\left(1+\frac{B(0) e^{(1-\gamma) r t}}{(1-\nu) K-B(0)}\right)=\frac{(1-v) K B(0) e^{(1-\gamma) r t}}{(1-v) K-B(0)} .
$$

Now, solving for $B(t)$, from the preceding equation, we obtain

$$
B(t)=\frac{(1-\nu) K B(0) e^{(1-\nu) r t}}{((1-\nu) K-B(0))+B(0) e^{(1-v) r t}} .
$$

In Section 7, using numerical simulations, we will analyze the impact of order of new fractional derivative and 
rank of control measure on the behavior of solutions of the population under the study.

\section{Numerical Simulations}

In this section, the numerical simulations of the solutions obtained using fractional derivative are carried out. The parameters values used in the simulations are taken in reference to [15]:

$$
\begin{aligned}
\tau & =548 \frac{\text { people }}{\text { day }}, \\
\mu & =\frac{0.0000548}{\text { day }}, \\
v & =\frac{0.033}{\text { day }} \\
r & =0.004, \\
\beta_{c} & =1.7 \times 10^{-8}, \\
\beta & =1.48 \times 10^{-8}, \\
\beta_{h} & =4 \times 10^{-10}, \\
\alpha & =0.001, \\
\omega & =\frac{0.2}{\text { day }}, \\
\varphi & =6 \times 10^{-6}, \\
K & =1000000, \\
0 & =10000 .
\end{aligned}
$$

\section{Result and Discussion}

In this study, we have investigated the behavior of population based on the new fractional-order derivative developed in [16]. In Figure 2, the forward bifurcation behavior of only HIV model is simulated. The forward bifurcation behavior of the only HIV model shows that the disease-free equilibrium is globally asymptotically stable if $R_{0}<1$ and unstable if $R_{0}>1$. However, endemic equilibrium is globally asymptotically stable whenever $R_{0}>1$ and unstable if $R_{0}<1$. The results obtained from numerical simulations described in Figure 3 show that increasing the fractional order increases the behavior of population toward controlling the infection. Also, the memory effects toward the invading disease are less whenever order of fractional derivative is near 0 but higher whenever the order of fractional derivative is near 1 . In Figure 4, the dynamics of $V$. cholerae concentration is simulated. The size of $V$. cholerae concentration in the environment increases significantly whenever intrinsic growth rate increases.

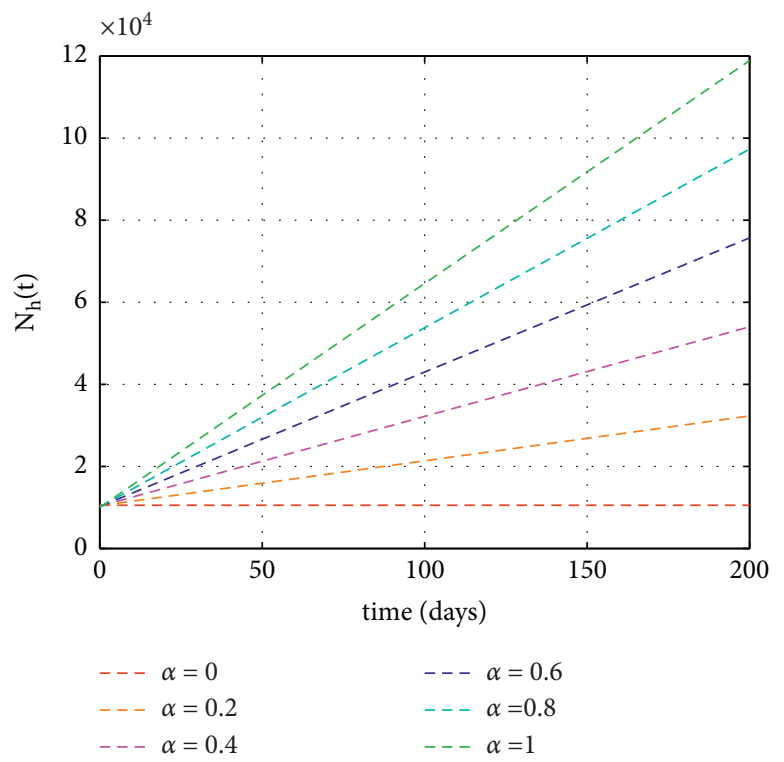

FIgURE 3: Simulation of dynamics of total human population size with order of derivative.

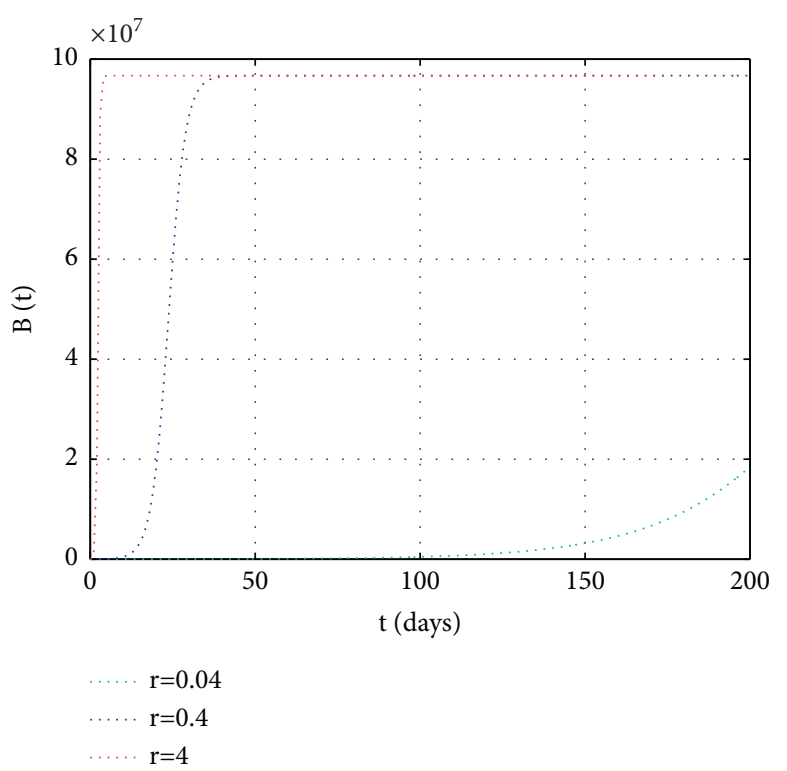

FIgure 4: Dynamics of $V$. cholerae concentration in the environment with different intrinsic growth rates.

\section{Conclusion}

The order of new fractional derivative is directly related to the behavior population toward the disease invading the population. The higher order of fractional derivative depicts that memory of population toward the disease increase so that the disease is controlled with applying possible controlling strategies and increasing population awareness toward HIV and cholera infections. The progression of the disease is described by classical derivative normally. Based on simulated forward bifurcation, the stability behavior of the steady state of the only HIV model is determined as 
stable or unstable. However, as the order of fractional derivative approaches to, 1 the number of infection-free population increases with time.

\section{Data Availability}

No data were used to support the findings of the study.

\section{Disclosure}

This study is a part of a Ph. D. thesis at Wollega University.

\section{Conflicts of Interest}

The authors declare that there are no conflicts of interest in the publication of this manuscript.

\section{Acknowledgments}

The first author would like to thank Hawasaa College of Teacher Education, Ministry of Science and Higher Education and Wollega University for their support to join PhD program and continue in research work.

\section{References}

[1] K. R. Cheneke, K. P. Rao, and G. K. Edessa, "Bifurcation and stability analysis of HIV transmission model with optimal control," Journal of Mathematics, vol. 2021, Article ID 7471290, 14 pages, 2021.

[2] O. M. Ogunlaran and S. C. O. Noutchie, "Mathematical model for an effective management of HIV infection," BioMed Research International, vol. 2016, Article ID 4217548, 6 pages, 2016.

[3] A. J. Arenas, G. González-Parra, J. J. Naranjo, M. Cogollo, and N. D. L. Espriella, "Mathematical analysis and numerical solution of a model of HIV with a discrete time delay," Mathematics, vol. 9, no. 3, p. 257, 2021.

[4] S. Mushayabasa and C. P. Bhunu, "Is HIV infection associated with an increased risk for cholera? insights from a mathematical model," Biosystems, vol. 109, no. 2, pp. 203-213, 2012.

[5] T. K. Ayele, E. F. D. Goufo, and S. Mugish, "Mathematical modeling of HIV/AIDS with optimal control: a case study in Ethiopia," Results in Physics, vol. 26, 2021.

[6] X. Cao, A. K. Roy, F. A. Basir, and P. K. Roy, "Global dynamics OF HIV infection with two disease transmission routes - a mathematical model," Communications in Mathematical Biology and Neuroscience, vol. 2020, p. 16, 2020.

[7] M. Bachraoui, K. Hattaf, and N. Yousfia, "Global stability of a fractional order HIV infection model with cure of infected cells in eclipse stage," Revue Africaine de la Recherche en Informatique et Mathématiques Appliquées, 2017.

[8] C. T. Codeço, "Endemic and epidemic dynamics of cholera: the role of the aquatic reservoir," BMC Infectious Diseases, vol. 1, p. 1, 2001.

[9] A. P. Lemos-Paião, C. J. Silva, and D. F. M. Torres, "A cholera mathematical model with vaccination and the biggest outbreak of world's history," AIMS Mathematics, vol. 3, no. 4, pp. 448-463, 2019.

[10] S. Liao, "Stability analysis and application of a mathematical cholera model," Mathematical Biosciences and Engineering, vol. 8, no. 3, pp. 733-752, 2011.
[11] F. Nyabadza, J. M. Aduamah, and J. Mushanyu, "Modelling cholera transmission dynamics in the presence of limited resources," BMC Research Notes, vol. 12, pp. 1-8, 2019.

[12] C. Yang and J. Wang, "On the intrinsic dynamics of bacteria in waterborne infections," Mathematical Biosciences, vol. 296, pp. 71-81, 2018.

[13] S. D. Hove-musekwa, F. Nyabadza, C. Chiyaka, and P. Das, "Modelling and analysis of the effects of malnutrition in the spread of cholera," Mathematical and Computer Modelling, vol. 53, no. 9-10, pp. 1583-1595, 2011.

[14] J. Lin, R. Xu, and X. Tian, "Transmission dynamics of cholera with hyperinfectious and hypoinfectious vibrios: mathematical modelling and control strategies," Mathematical Biosciences and Engineering, vol. 16, no. 5, pp. 4339-4358, 2019.

[15] K. R. Cheneke, K. P. Rao, and G. K. Edessa, "Application of a new generalized fractional derivative and rank of control measures on cholera transmission dynamics," International Journal of Mathematics and Mathematical Sciences, vol. 2021, Article ID 2104051, 9 pages, 2021.

[16] K. Hattaf, "A new generalized definition of fractional derivative with non-singular," Kernel, Computation, vol. 8, 2020.

[17] P. V. D. Driessche, "Reproduction numbers of infectious disease models," Infectious Disease Modelling, vol. 2, no. 3, pp. 288-303, 2017.

[18] S. Sharma and G. P. Samanta, "Analysis of a chlamydia epidemic model," Journal of Biological Systems, vol. 22, no. 4, pp. 1-32, 2014.

[19] S. K. Biswas, U. Ghosh, and S. Sarkar, "Mathematical model of zika virus dynamics with vector control and sensitivity analysis," Infectious Disease Modelling, vol. 5, pp. 23-41, 2020.

[20] J. O. Akanni, S. Olaniyi, and F. O. Akinpelu, "Global asymptotic dynamics of a nonlinear illicit drug use system," Journal of Applied Mathematics and Computing, vol. 66, 2020.

[21] E. A. Bakare and S. Hoskova-Mayerova, "Optimal control analysis of cholera dynamics in the presence of asymptotic transmission," Axioms, vol. 10, 2021.

[22] S. Sharma and G. P. Samanta, "Dynamical behavior of an HIV/AIDS epidemic model," Differential Equations and Dynamical Systems, vol. 22, 2013.

[23] K. Hattaf, A. A. Lashari, Y. Louartassi, and N. Yousf, "A delayed SIR epidemic model with general incidence rate," Electronic Journal of Qualitative Theory of Differential Equations, vol. 3, pp. 1-9, 2013.

[24] S. Khajanchi, "Uniform persistence and global stability for a brain tumor and immune system interaction," Biophysical Reviews and Letters, vol. 12, no. 4, pp. 1-22, 2017.

[25] S. Khajanchi and S. Banerjee, "Quantifying the role of immunotherapeutic drug T11 target structure in progression of malignant gliomas: mathematical modeling and dynamical perspective," Mathematical Biosciences, vol. 289, pp. 69-77, 2017.

[26] F. A. Basir, S. Raya, and E. Venturino, "Role of media coverage and delay in controlling infectious diseases: a mathematical model," Applied Mathematics and Computation, vol. 337, 2018.

[27] P. K. Roy, S. Saha, and F. A. Basir, "Effect of awareness programs in controlling the disease HIV/AIDS: an optimal control theoretic approach," Advances in Difference Equations, vol. 217, 2015.

[28] K. H. Hntsa and B. N. Kahsay, "Analysis of cholera epidemic controlling using mathematical modeling," International Journal of Mathematics and Mathematical Sciences, vol. 2020, Article ID 7369204, 13 pages, 2020. 
[29] A. S. Waziri, E. S. Massawe, and O. D. Makinde, "Mathematical modelling of HIV/AIDS dynamics with treatment and vertical transmission," Applied Mathematics, vol. 2, no. 3, pp. 77-89, 2012.

[30] H. Abboubakar, J. C. Kamgang, and D. Tieudjo, "Bifurcation thresholds and optimal control in transmission dynamics of arboviral diseases," 2016, https://arxiv.org/abs/1601.02510v1.

[31] S. F. Abimbade, S. Olaniyi, O. A. Ajala, and M. O. Ibrahim, "Optimal control analysis of a tuberculosis model with exogenous re-infection and incomplete treatment," Optimal Control Applications and Methods, vol. 41, pp. 1-20, 2020.

[32] S. Olaniyi, M. Lawal, and O. S. Obabiyi, "Stability and sensitivity analysis of a deterministic epidemiological model with pseudo-recovery," IAENG International Journal of Applied Mathematics, vol. 46, no. 2, 2016.

[33] B. Seidu and O. D. Makinde, "Optimal control of HIV/AIDS in the workplace in the presence of careless individuals," Computational and Mathematical Methods in Medicine, vol. 2014, Article ID 831506, 19 pages, 2014.

[34] I. S. Onah, O. C. Collins, P. G. U. Madueme, and G. C. E. Mbah, "Dynamical system analysis and optimal control measures of lassa fever disease model," International Journal of Mathematics and Mathematical Sciences, vol. 2020, Article ID 7923125, 18 pages, 2020.

[35] Fatmawati and H. Tasman, "An optimal treatment control of TB-HIV coinfection," International Journal of Mathematics and Mathematical Sciences, vol. 2016, Article ID 8261208, 11 pages, 2016.

[36] K. Hattaf, "Stability of fractional differential equations with new generalized Hattaf fractional derivative," Mathematical Problems in Engineering, vol. 2021, Article ID 8608447, 7 pages, 2021. 